\title{
Avaliação da migração do espaçador intersomático (cage) em pacientes submetidos à artrodese lombar pela técnica TLIF
}

Rodrigo Rezende', Fernando Junqueira de Faria², Igor Pellucci Pinto², José Lucas Batista Júnior ${ }^{3}$, Chárbel Jacob Júnior, Igor Machado Cardoso ${ }^{3}$

Hospital Santa Casa de Misericórdia de Vitória, Vitória, ES, Brasil.

\section{RESUMO}

Objetivo: Avaliar a migração do espaçador intersomático (cage) em pacientes submetidos à artrodese lombar pela técnica transforaminal (TLIF). Método: Estudo retrospectivo de 27 pacientes submetidos à técnica TLIF nos níveis L4-L5, L5-S1 ou L4-L5 e L5-S1 para tratamento de patologia degenerativa da coluna lombar entre julho de 2009 e julho de 2011. Os dados referentes à migração do cage foram obtidos a partir da análise de imagens radiográficas da coluna lombar nas incidências anteroposterior $(A P)$ e perfil nos momentos pré e pós-operatório com 1, 6 e 12 meses. O valor utilizado como critério de migração do cage foi determinado pelo deslocamento anterior ou posterior maior ou igual a $2 \mathrm{~mm}$ quando comparado a exame radiográfico pré e pós-operatório. Resultados: Foi inserido um total de 36 cages. Dos 27 pacientes avaliados, 15 (55,5\%) apresentavam algum tipo de migração do cage em 4 (14,8\%) a migração foi para anterior e em 11 (40,7\%) a migração foi para posterior. Conclusão: Encontramos migração em $55,5 \%$ do total de pacientes, e em $40,7 \%$ a migração foi posterior, porém sem necessidade de novas intervenções cirúrgicas.

\section{PALAVRAS-CHAVE}

Artrodese, fixadores internos, complicações pós-operatórias.

\section{ABSTRACT}

Migration assessment of interbody spacer in patients undergoing lumbar arthrodesis by technique of TLIF

Objective: To evaluate the cage migration in transforaminal lumbar interbody fusion (TLIF). Method: We retrospectively reviewed the records of 27 patients who had been diagnosed with degenerative lumbar disease, and who had undergone a transforaminal lumbar interbody fusion at L4-L5, L5-S1, and L4L5/L5-S1 between July 2009 and July 2011. All data regarding the cage migration was obtained from preoperative and postoperative radiographs including standing anteroposterior $(A P)$ and lateral. Clinical and radiographic assessment was performed at 1, 6 and 12 months after surgery. Cage migration was identified if cage moved posteriorly $2 \mathrm{~mm}$ or more compared with previous radiographs. Results: 36 cages were inserted. Cage migration was found in 15 of 27 patients (55,5\%). Four cases of anterior displacement $(14,8 \%)$ and 11 cases of posterior displacement $(40,7 \%)$ were found. Conclusion: The rate of cage migration was $55.5 \%$. In addition, $40.7 \%$ of posterior displacement was found but without further surgical intervention.

\section{KEYWORDS}

Arthrodesis, internal fixators, postoperative complications.

Chefe do Grupo de Coluna Vertebral do Hospital Santa Casa de Misericórdia de Vitória, Vitória, ES, Brasil.

2 Graduando do curso de Medicina da Escola Superior de Ciências da Saúde da Santa Casa de Misericórdia (Emescam), Vitória, ES, Brasil.

3 Ortopedista, especialista em Coluna, médico-assistente do Grupo de Coluna Vertebral do Hospital Santa Casa de Misericórdia de Vitória, Vitória, ES, Brasil. 


\section{Introdução}

A lombalgia, ou lombociatalgia, acomete de $67 \%$ a $84 \%$ da população de países industrializados em algum momento da vida, ${ }^{1}$ e a instabilidade vertebral associada ou não à doença discal se caracteriza como um importante fator etiológico nessa patologia.

Nos casos de pacientes portadores de lombalgia refratária ao tratamento conservador e após o diagnóstico preciso e criterioso de instabilidade, existe indicação de se realizar artrodese vertebral, que consiste na fusão óssea entre duas vértebras.

Historicamente, a primeira artrodese lombar foi descrita, em 1911, por Russel Hibbs e Fred Albee para prevenção de deformidade progressiva da coluna vertebral, sendo realizada in situ com enxerto autólogo. ${ }^{2-4} \mathrm{Na}$ década de 1970, popularizou-se a utilização de parafusos pediculares nas artrodeses vertebrais, ${ }^{5,6} \mathrm{e}$ aparecimento desses novos materiais contribuiu para diminuir a taxa de pseudoartrose encontrada em fusões in situ. ${ }^{7}$

Apesar de a artrodese posterolateral com utilização de parafusos pediculares ter representado um grande avanço na cirurgia de coluna, complicações como pseudoartrose, soltura e quebra do material de síntese ainda persistiam. Por isso, visando diminuir cada vez mais essas complicações, a partir de 1998 associou-se a artrodese posterolateral à artrodese anterior, podendo esta ser realizada pela técnica ALIF (fusão lombar via anterior), PLIF (fusão lombar via posterior) e TLIF (fusão lombar via transforaminal), porém essas técnicas não são isentas de complicações e podem levar à necessidade de nova intervenção cirúrgica se não forem realizadas adequadamente.

As complicações mais frequentes descritas com a técnica de TLIF são: falha no alívio da dor, infecção, lesão vascular, lesão neurológica, pseudoartrose e migração pós-operatória do cage.

Entre as complicações citadas, a migração do cage talvez seja a mais temida pelos cirurgiões, pois, quando migrado para posterior, pode comprimir as estruturas nervosas, levando à recidiva dos sintomas pré-operatórios e à necessidade de uma nova intervenção cirúrgica - nem sempre fácil por causa do posicionamento do espaçador intersomático -, podendo causar lesões neurológicas irreversíveis no momento de sua retirada ou reposicionamento.

Devido à falta de trabalhos na literatura que estabeleçam critérios quantitativos de migração do cage em cirurgia de coluna, ${ }^{8}$ objetivamos, nesta pesquisa, avaliar a migração do cage em pacientes submetidos à artrodese lombar pela técnica TLIF.

\section{Materiais e métodos}

Foi realizado um estudo clínico e radiográfico, retrospectivo, envolvendo inicialmente 72 pacientes submetidos à fusão intersomática lombar transforaminal, dos quais 27 preencheram os critérios de seleção, no período compreendido entre julho de 2009 e julho de 2011 (Figura 1), autorizado previamente pelo Comitê de Ética e Pesquisa da Escola Superior de Ciências da Saúde da Santa Casa de Misericórdia de Vitória sob o parecer número 84407.

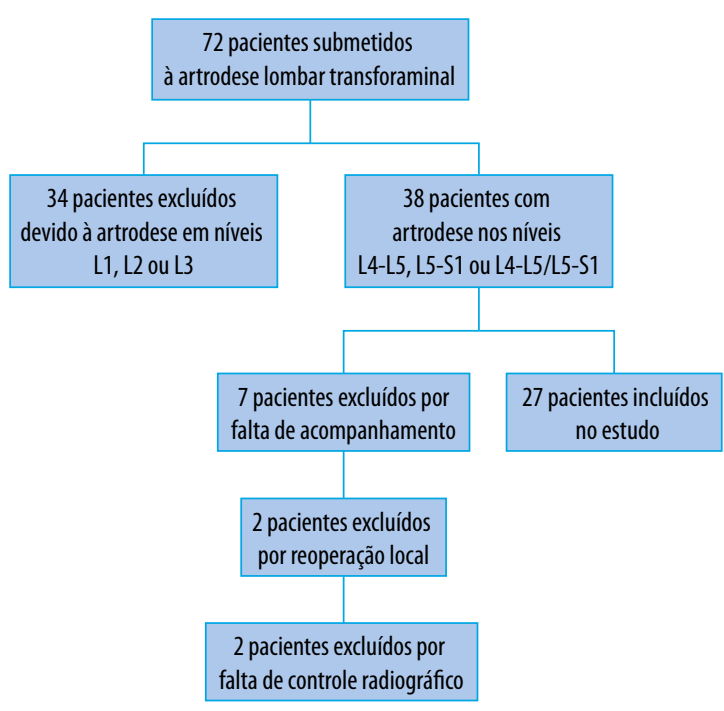

Figura 1 - Diagrama para inclusão no estudo.

Foram incluídos no presente estudo pacientes refratários ao tratamento conservador, de ambos os sexos, sem distinção de idade ou raça, portadores de patologias degenerativas, com instabilidade da coluna lombar nos níveis L4-L5, L5-S1 ou L4-L5/L5-S1, diagnosticadas por meio de exame clínico, radiográfico e ressonância magnética. Consideramos como critério de instabilidade vertebral a angulação do platô vertebral maior ou igual a $10^{\circ}$ (dez graus) ou escorregamento vertebral maior que $3 \mathrm{~mm}$ (três milímetros) aferidos na radiografia dinâmica, como definido por Dupuis et al. ${ }^{9}$

Os critérios de exclusão foram pacientes submetidos a tratamento conservador adequado com melhora, pacientes submetidos à nova intervenção cirúrgica local, pacientes submetidos à artrodese lombar envolvendo os níveis L1, L2 ou L3 e aqueles cujo acompanhamento pós-operatório não foi realizado pela mesma equipe cirúrgica responsável.

Os dados referentes às complicações foram obtidos a partir da revisão dos protocolos estabelecidos pelo grupo, assim como da análise de imagens radiográficas da coluna lombar nas incidências anteroposterior (AP) e perfil nos momentos pré e pós-operatório tardio. A análise pós-operatória foi realizada com radiografias nas primeiras 24 horas de cirurgia, assim como em avaliações sucessivas com um, seis e 12 meses por equipe 
cirúrgica em sessões clínicas, formada por quatro médicos ortopedistas e especialistas em cirurgia da coluna.

Os valores obtidos pela análise de imagens radiográficas foram alcançados por meio de software (Surgimap Spine $^{\circledR}$ versão 1.1.2.169, 2011-2012), medindo-se a distância entre o ponto mais posterior do marcador radiopaco do cage e uma linha tangente à cortical posterior do corpo vertebral situado imediatamente acima do espaçador intersomático. A distância encontrada deveria ser perpendicular à linha referencial supracitada e foi mensurada em milímetros (Figura 2).

O valor utilizado como critério de migração do cage foi determinado pelo deslocamento anterior ou posterior maior ou igual a $2 \mathrm{~mm}$ comparado a exame radiográfico prévio.

Ao final, todos os dados foram transcritos em protocolo desenvolvido pelo serviço de cirurgia da coluna do referido hospital, tabulados no software Microsoft Excel $^{\circledR} 2010$ (v.14) incluído no Office ${ }^{\circledR} 2010$ e salvos em banco de dados.
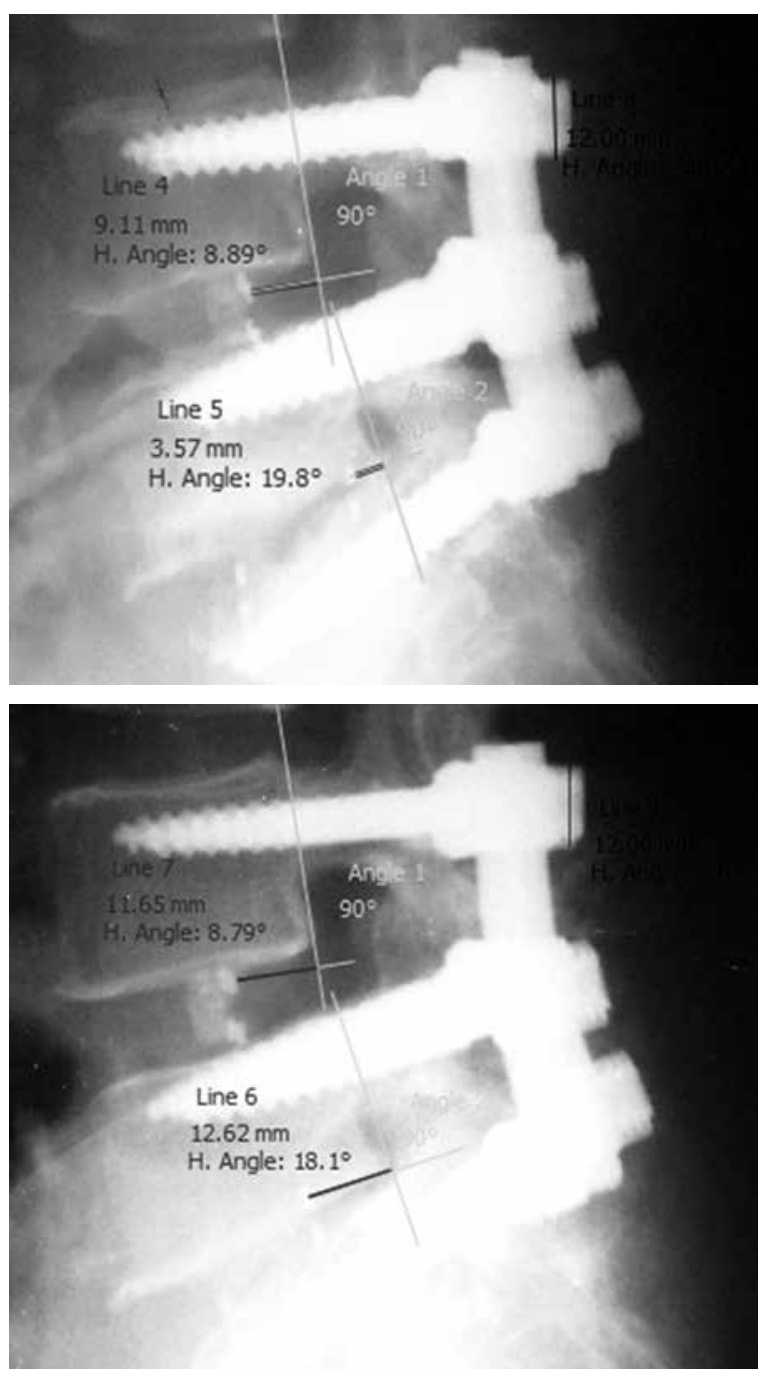

Figura 2 - Método utilizado para avaliar a migração do cage.

\section{Resultados}

Dos 27 pacientes envolvidos no estudo, 14 (51,85\%) pertenciam ao sexo masculino. A média de idade para ambos os sexos foi de 48,4 anos, com variação mínima e máxima de 23 e 68 anos, respectivamente. O período médio de sintomatologia dos pacientes previamente à cirurgia foi de 24,37 meses.

Do total da amostra estudada, 15 pacientes $(55,55 \%)$ foram submetidos à artrodese em um nível, L4-L5 ou L5-S1, e 12 pacientes (44,45\%), em dois níveis vertebrais, L4-L5/L5-S1 (Tabela 1).

\begin{tabular}{|c|c|c|c|c|}
\hline \multicolumn{5}{|c|}{ Tabela 1 - Níveis de artrodese } \\
\hline $\begin{array}{l}\text { Nível de } \\
\text { artrodese }\end{array}$ & L4-L5 & L5-S1 & $\begin{array}{c}\text { L4-L5/ } \\
\text { L5-S1 }\end{array}$ & Total \\
\hline $\mathrm{N}$ & 6 & 9 & 12 & 27 \\
\hline
\end{tabular}

O tempo de cirurgia variou de 120 a 240 minutos, com uma média de 176 minutos nas intervenções de um ou dois níveis.

Apresentamos uma taxa de complicações intraoperatórias de $14,81 \%$, o que corresponde a um total de quatro complicações em pacientes distintos. As complicações observadas foram sangramento devido a lesão de vasos epidurais, lesão dural, quebra do cage durante sua introdução e dor associada a alterações parestésicas em membros inferiores.

O tempo médio de internação pós-operatória foi de 5,4 dias, e o menor tempo de internação foi de três dias e o maior tempo, de oito dias.

Foi utilizado um total de 36 cages; em 18 indivíduos foi inserido apenas um cage e em nove foram inseridos dois cages. Dos 27 pacientes avaliados, 15 (55,5\%) apresentavam algum tipo de migração do cage, e em quatro $(14,8 \%)$ a migração foi para anterior e em $11(40,7 \%)$ a migração foi para posterior (Tabela 2).

\begin{tabular}{|c|c|c|c|}
\hline $\begin{array}{l}\text { Total de } \\
\text { pacientes com } \\
\text { cages inseridos }\end{array}$ & $\begin{array}{c}\text { Total de } \\
\text { pacientes com } \\
\text { migrações }\end{array}$ & $\begin{array}{l}\text { Pacientes com } \\
\text { migração } \\
\text { anterior }\end{array}$ & $\begin{array}{c}\text { Pacientes com } \\
\text { migração } \\
\text { posterior }\end{array}$ \\
\hline 27 (100\%) & $15(55,5 \%)$ & $4(14,8 \%)$ & $11(40,7 \%)$ \\
\hline
\end{tabular}

Quando analisamos os resultados específicos dos 18 pacientes que receberam apenas um cage, observamos que oito $(44,5 \%)$ apresentaram algum tipo de migração do espaçador; desses, cinco $(27,8 \%)$ apresentaram migração posterior e três $(16,7 \%)$ apresentaram migração anterior (Tabela 3). 


\begin{tabular}{lccc}
\hline \multicolumn{4}{c}{ Tabela 3 - Pacientes com inserção de um cage } \\
\hline $\begin{array}{l}\text { Total de } \\
\text { pacientes que } \\
\text { receberam } \\
\mathbf{1} \text { cage }\end{array}$ & $\begin{array}{c}\text { Total de } \\
\text { pacientes com } \\
\text { migração }\end{array}$ & $\begin{array}{c}\text { Pacientes com } \\
\text { migração } \\
\text { anterior }\end{array}$ & $\begin{array}{c}\text { Pacientes com } \\
\text { migração } \\
\text { posterior }\end{array}$ \\
\hline $18(100 \%)$ & $8(44,5 \%)$ & $3(16,7 \%)$ & $5(27,8 \%)$ \\
\hline
\end{tabular}

Dos nove indivíduos que receberam dois cages, sete $(77,8 \%)$ apresentaram algum tipo de migração do espaçador; desses, cinco pacientes $(55,6 \%)$ apresentaram migração em apenas um cage e dois $(22,2 \%)$ apresentaram migração em ambos os cages (Tabela 4).

\begin{tabular}{|c|c|c|c|}
\hline \multicolumn{4}{|c|}{ Tabela 4 - Pacientes com inserção de dois cages } \\
\hline $\begin{array}{l}\text { Total de } \\
\text { pacientes que } \\
\text { receberam } \\
2 \text { cages }\end{array}$ & $\begin{array}{c}\text { Total de } \\
\text { pacientes com } \\
\text { migração }\end{array}$ & $\begin{array}{c}\text { Pacientes com } \\
\text { migração de } \\
\text { apenas } 1 \text { cage }\end{array}$ & $\begin{array}{l}\text { Pacientes com } \\
\text { migração } \\
\text { de } 2 \text { cages }\end{array}$ \\
\hline $9(100 \%)$ & $7(77,8 \%)$ & $5(55,6 \%)$ & $2(22,2 \%)$ \\
\hline
\end{tabular}

\section{Discussão}

As artrodeses da coluna lombar têm sido utilizadas para tratamento de várias condições patológicas, no entanto os procedimentos cirúrgicos não são isentos de complicações.

Com o advento da instrumentação pedicular, a taxa de fusão se tornou mais elevada, mas não atingindo ainda níveis aceitáveis como apresentado por Harms e Jeszensky ${ }^{10} \mathrm{e}$ Whitecloud et al. ${ }^{11}$ Glazer et al. ${ }^{12}$ relatam que $80 \%$ da carga transmitida para o segmento lombar passam pela coluna anterior, enquanto $20 \%$ passam pela coluna posterior. Devido a esse fato, as artrodeses anteriores associadas às posterolaterais vêm ganhando cada vez mais espaço entre os cirurgiões.

Em nosso trabalho, todos os pacientes foram abordados pela via posterior, conforme técnica cirúrgica da fusão intersomática lombar por abordagem transforaminal $\left(360^{\circ}\right)$. A técnica TLIF, popularizada por Harms e Jeszensky ${ }^{10} \mathrm{em} 1998$, permite amplo acesso ao espaço intervertebral através da abertura de um neuroforame, reduzindo o número de complicações quando comparada a outras técnicas. ${ }^{13}$

A incidência geral de eventos adversos ou complicações em cirurgias da coluna vertebral é desconhecida, ${ }^{14}$ e muitos fatores contribuem para essa divergência de resultados, uma vez que nem mesmo a definição de complicação é consenso. ${ }^{14,15}$

Por esse motivo, resolvemos em nosso trabalho analisar apenas a presença ou não da migração do cage nas artrodeses intersomáticas pela técnica TLIF, talvez por essa ser a complicação mais temida pelos cirurgiões de coluna.
Entretanto, avaliar a migração de cage como complicação se torna tarefa difícil, uma vez que não se encontram critérios quantitativos na literatura que definam a migração do espaçador. Em 2005, Chen et al. ${ }^{8}$ apresentaram seu estudo de migração de cage em pacientes tratados pela técnica PLIF e na ocasião utilizaram como medida-padrão o deslocamento maior ou igual a $2 \mathrm{~mm}$. Abbushi et al., em 2009, avaliando a migração do cage influenciada pelo posicionamento e o tipo de espaçador em pacientes tratados também pela técnica PLIF, utilizaram como critério de migração o valor de $3 \mathrm{~mm} .{ }^{16}$

Por não existirem na literatura estudos que revelem critérios quantitativos que definam a migração do espaçador intersomático, tampouco relacionados à técnica TLIF, utilizamos neste trabalho os valores estabelecidos por Chen et al. ${ }^{8}$, ou seja, $2 \mathrm{~mm}$, como presença de migração.

Encontramos migração em 17 cages de um total de 36 inseridos. Essas migrações ocorreram em $55,5 \%$ dos pacientes submetidos à artrodese transforaminal em um ou dois níveis. Desse total, 14,8\% foram de migrações anteriores, o que não gera nenhum risco ao paciente, $\mathrm{e}$ 40,7\% foram de migrações para posterior. Quando comparamos nossa taxa de migração com outros trabalhos - Chen et al. ${ }^{8}$ revelaram taxa de $16,7 \%$ e Abbushi et al..$^{16}$ apresentaram taxa de $30 \%$ de migração -, ela permanece elevada, porém em nenhum caso foi necessária uma reabordagem cirúrgica.

A elevada taxa de migração encontrada no presente estudo talvez se deva ao fato de utilizarmos como critério de migração o valor de $2 \mathrm{~mm}$, ou seja, uma margem pequena de deslocamento, que pode ocorrer por causa da acomodação do material no espaço intersomático.

Por fim, julgamos que a utilização da técnica TLIF se apresenta como alternativa viável nas artrodeses vertebrais, permitindo elevada taxa de consolidação óssea quando comparada a outras técnicas, devendo o cirurgião estar sempre alerta para o posicionamento ideal do espaçador intersomático, a fim de evitar complicações neurológicas indesejadas.

\section{Conclusão}

Encontramos migração em $55,5 \%$ do total de pacientes, e em 40,7\% a migração foi posterior, porém sem necessidade de novas intervenções cirúrgicas.

\section{Conflito de interesses}

Os autores declaram inexistência de conflito de interesses na realização deste trabalho. 


\section{Referências}

1. Fourney DR, Andersson G, Arnold PM, Dettori J, Cahana A, Fehlings MG, et al. Chronic low back pain: a heterogeneous condition with challenges for an evidence-based approach. Spine (Phila Pa 1976). 2011;36(Suppl 21):1-9.

2. Moon MS. Development in the management of tuberculosis of the spine. Cur Orthop. 2006;20:132-40.

3. Heary RF, Madhavan K. The history of spinal deformity. Neurosurgery. 2008;63(Suppl 3):5-15.

4. Young PM, Berquist TH, Bancroft LW, Peterson JJ. Complications of spinal instrumentation. Radiographics. 2007;27(3):775-89.

5. Hu SS, Pashman RS. Spinal instrumentation. Evolution and state of the art. Invest Radiol. 1992;27(8):632-47.

6. Kabins MB, Weinstein JN. The history of vertebral screw and pedicle screw fixation. lowa Orthop J. 1991;11:127-36.

7. Etminan M, Girardi FP, Khan SN, Cammisa FP Jr. Revision strategies for lumbar pseudarthrosis. Orthop Clin North Am. 2002;33(2):381-92.

8. Chen L, Yang H, Tang T. Cage migration in spondylolisthesis treated with posterior lumbar interbody fusion using BAK cages. Spine (Phila Pa 1976). 2005;30(19):2171-5.

9. Dupuis PR, Yong-Hing K, Cassidy JD, Kirkaldy-Willis WH. Radiologic diagnosis of degenerative lumbar spinal instability. Spine (Phila Pa 1976). 1985;10(3):262-76.

10. Harms JG, Jeszensky D. The unilateral, transforaminal approach for posterior lumbar interbody fusion. Orthop Traumatol. 1998;6:88-99.

11. Whitecloud TS 3rd, Roesch WW, Ricciardi JE. Transforaminal interbody fusion versus anterior-posterior interbody fusion of the lumbar spine: a financial analysis. J Spinal Disord. 2001;14(2):100-3.

12. Glazer PA, Colliou O, Lotz JC, Bradford DS. Biomechanical analysis of lumbosacral fixation. Spine (Phila Pa 1976). 1996;21(10):1211-22.

13. Faundez AA, Schwender JD, Safriel Y, Gilbert TJ, Mehbod AA, Denis F, et al. Clinical and radiological outcome of anterior-posterior fusion versus transforaminal lumbar interbody fusion for symptomatic disc degeneration: a retrospective comparative study of 133 patients. Eur Spine J. 2009;18(2):203-11.

14. Nasser R, Yadla S, Maltenfort MG, Harrop JS, Anderson DG, Vaccaro AR, et al. Complications in spine surgery. J Neurosurg Spine. 2010;13(2):144-57.

15. Ratliff JK, Lebude B, Albert T, Anene-Maidoh T, Anderson G, Dagostino P, et al. Complications in spinal surgery: comparative survey of spine surgeons and patients who underwent spinal surgery. J Neurosurg Spine. 2009;10(6):578-84.

16. Abbushi A, Cabraja M, Thomale UW, Woiciechowsky C, Kroppenstedt SN. The influence of cage positioning and cage type on cage migration and fusion rates in patients with monosegmental posterior lumbar interbody fusion and posterior fixation. Eur Spine J. 2009;18(11):1621-8.

\section{Endereço para correspondência}

Rodrigo Rezende

Serviço de Coluna Vertebral do Hospital

Santa Casa de Misericórdia de Vitória

Rua Doutor João Santos Neves, 143, Vila Rubim

29018-180 - Vitória, ES, Brasil

Telefone: (27) 3389-0305

E-mail: rezenderodrigo@hotmail.com 